\title{
¿Qué nos pueden enseñar los estoicos sobre el valor de los valores? *
}

\author{
OSVALDO GUARIGLIA \\ Universidad de Buenos Aires-CONICET
}

$\S 1$. En un extenso e interesante pasaje del libro Contra los eticistas, Sexto Empírico nos expone la doctrina estoica mediante la cual se hace una división entre el sentido de los términos que expresan «bondad», «indiferencia» $\mathrm{y}$ «preferencia» en el ámbito moral. A continuación cito las partes más relevantes de esta exposición:

Contra los eticistas, 59-63, «[Los estoicos] suponen que el término "indiferente" se dice de tres maneras distintas: en un sentido, se aplica a aquello que no provoca ni atracción ni repulsión, como, por ejemplo, que el número de [...] los cabellos en nuestra cabeza sea par o impar; en otro sentido, se aplica a aquello que despierta atracción o repulsión pero no más para una que para otra cosa del mismo género, como, por ejemplo, en el caso de dos monedas de un dracma, que no se distinguen entre sí ni por su cuño ni por su brillo. [...] En tercer y último lugar, dicen que "indiferente" es aquello que no contribuye ni a la felicidad ni a la infelicidad, e indiferente en este sentido dicen que son la salud y la enfermedad y todo lo referente al cuerpo y la mayoría de las cosas exteriores, porque ellas no tienden ni a la felicidad ni a la infelicidad. En efecto, aquello que es posible de ser usado bien o mal, será indiferente, y mientras que uno siempre usa bien la virtud y mal el vicio, uno puede usar la salud y las cosas del cuerpo unas veces bien y otras mal, por lo cual éstas serán indiferentes. También agregan que algunas de las cosas indiferentes son preferidas, otras postergadas y otras más, por último, ni preferidas ni postergadas: "preferidas" son, pues, aquellas que tienen suficiente valor; "postergadas", aquellas otras que tienen suficiente disvalor; por último, ni preferidas ni postergadas son cuestiones como, por ejemplo, extender o contraer el dedo. Los estoicos ordenan dentro de lo preferido la salud, la fortaleza, la belleza, la riqueza, la reputación, y todo lo semejante; dentro de lo postergado, la enfermedad, la pobreza, el dolor, y todo lo semejante.»

Los dos pilares que sostienen la ética estoica fueron las dos tesis siguientes: 1) moralmente buenas son solamente las acciones de acuerdo con la virtud y moralmente malas las acciones contrarias a éstas; y 2) el ejercicio de la virtud moral, y solamente él, constituye y garantiza una vida feliz. Como consecuencia de estas dos tesis, todos los demás bienes, como los externos o los que corresponden a la salud y la configuración del cuerpo, dejaron de ser considerados «bienes» en sentido estricto para convertirse en «indiferentes». En realidad, se trató de una distinción que introducía una diferencia de grado más que una división categorial dentro de la clasificación aristotélica de los bienes, la cual solía distinguir entre bienes exteriores, del cuerpo y del alma, subordinando claramente los dos primeros a los últimos. Pese a ello, la restricción estoica del significado de «bondad» exclusivamente a los actos virtuosos, que a su vez provenían sólo del uso de la razón, fijó por primera vez de un modo neto los límites de la calificación moral, distinguiéndola de todo

* La presente versión de este trabajo reproduce con algunas enmiendas mi contribución a la Mesa redonda sobre «Ciencia, Tecnología y Valores», que tuvo lugar en Morelia, México, como parte del I Congreso Iberoamericano de la Ciencia y la Tecnología del 26 al 30 de septiembre de 2000 . 
aquello que dependía, en última instancia, de circunstancias o contingencias externas, sujetas a la variación fortuita de las causas del mundo natural o del azar.

Por cierto, la metafísica estoica que otorgaba una garantía firme a la prudencia del sabio, aseguraba que éste tenía acceso al lógos que gobernaba al universo y se guiaba por él en sus elecciones, de modo que sus juicios morales gozaban de una especie de infalibilidad. De este modo, la virtud en la conducta del sabio y la ley divina que regía el mundo estaban en una relación de correspondencia; como consecuencia, no cabían dudas, al menos para el sabio, sobre lo que era moralmente correcto o moralmente repudiable. A la inversa, fuera de estos rígidos márgenes se abría un ancho espacio para la acción que, en última instancia, se regía por «preferencias» razonablemente fundadas. A todas las acciones y estados de cosas del cuerpo o de las pertenencias exteriores, sea de orden intelectual, como la fama, o de orden material, como la riqueza, los estoicos los consideraron «indiferentes».

Como ya nos muestra el texto citado de Sexto Empírico, hay una diferencia clara entre tres niveles de indiferencia: $a$ ) las acciones o cosas que no provocan ni atracción ni repulsión; $b$ ) las que provocan genéricamente atracción (o repulsión), pero son indiferentes entre sí (ejemplo de las dos monedas semejantes); y, por último, c) aquellos estados del cuerpo o de las pertenencias que provocan atracción o repulsión natural. En efecto, dado que estos últimos se correspondían con un impulso o una repulsión en el agente, su satisfacción constituía lo que los estoicos llamaron «un acto debido». Como consecuencia, las cosas indiferentes obtuvieron un status ambiguo en la ética estoica, que dio lugar a numerosas controversias desde la antigüedad, especialmente con respecto a la relación entre, por un lado, los actos debidos o apropiados (kathêkonta) y, por el otro, los indiferentes de la especie «pre- feridos». En efecto, las fuentes nos reportan la existencia de un criterio para distinguir entre indiferentes valiosos y disvaliosos, que se basa en su función «según la naturaleza» o «contra la naturaleza».

Estobeo, II 79, 18-80, 13 (LS, 58C). «Unas cosas son según naturaleza, otras contra naturaleza, y otras no son contra naturaleza ni según naturaleza. Ahora bien, cosas de esta índole son según naturaleza: salud, fuerza, el buen funcionamiento de los órganos de los sentidos y cosas similares; contra naturaleza, en cambio, son cosas de este tipo: enfermedad, debilidad, una mutilación y cosas como éstas; [...] Y dicen que el argumento relativo a estas cuestiones se hace a partir de cosas primeras según naturaleza y contra naturaleza, ya que lo diferente y lo indiferente se encuentra entre lo que es dicho respecto de algo. Porque, afirman, aunque llamáremos indiferentes a las cosas corporales y a las cosas exteriores, afirmamos que ellas son indiferentes respecto al vivir con decoro (aquello en lo cual, precisamente, se da el vivir con felicidad), pero no, por Zeus, respecto al hecho de estar en concordancia con la naturaleza, ni en relación con el impulso (horme $\bar{e}$ ) ni con la repulsión (aphorme $\bar{e}$ ).»

Estobeo, II 82, 20-21. «Todas las cosas según naturaleza son objeto de aceptación (lēptá), en tanto que todas las cosas contrarias a la naturaleza no son objeto de aceptación.»

Estobeo, II 83, 10-11. «Todas las cosas según naturaleza tienen valor (axía) y todas las cosas contrarias a la naturaleza, disvalor (apaxía)» (traducción de M. Boeri).

Entre los indiferentes preferidos están, pues, 1) en primer lugar, aquellos que son según naturaleza y corresponden a un impulso. Por lo tanto, es de suponer que los estoicos entienden por éstos aquellas cosas hacia las que tendemos desde que nacemos o en nuestra primera infancia (alimento, abrigo, cuidado, etc.) comprendidas en su conjunto como medios de preservación de sí. Como se ve, no existe aquí reflexión o elección, sino meramente hor$m \bar{e}$, es decir, un impulso que precisamente es un «móvil» de la obtención del objeto exterior al que tiende. 2) En un nivel supe- 
rior se encuentran los indiferentes considerados valiosos, ya que aquí aparece un juicio que atribuye o niega una estimación a la cosa que se nos presenta como móvil del impulso. Esta estimación del objeto de la acción no es, aún, moral, pero tiene un carácter prescriptivo, a fin de ordenar convenientemente nuestros actos, considerados debidos con relación a la naturaleza (kathékonta: «aquello que una vez realizado comporta una justificación razonable» Estobeo, II 85, $13=$ SVF III, $494=$ LS, 59B). Estas reglas de comportamiento suelen expresarse como imperativos de conducta: «iharás esto!, ievitarás eso otro!», que están dirigidas al hombre común (es decir, no al sabio) a fin de que éste encuentre en ellas la ayuda necesaria para conducir su vida hasta que él mismo esté en condiciones de dirigirla (Séneca, Ep. 94, 50-51). 3) En el último nivel, encontramos aquellas cosas de acuerdo con la naturaleza que no solamente son el principio de los actos apropiados sino que constituyen, especialmente, la materia de los actos virtuosos (Plutarco, De comm. not. 23, 1069 $\mathrm{e}=S V F$ III, 491). De este modo, los actos debidos pasan a ser actos rectos (katórthoma), realizados a partir de una disposición del espíritu para seleccionar y resolverse por esa acción como un fin en sí misma, porque ésta constituye una manifestación de la virtud. Las cosas indiferentes como tales, por lo tanto, solamente tienen el valor que les confiere el ser producto de una elección (Plutarco, De comm. not. 26, $1071 \mathrm{a}-\mathrm{b})^{1}$.

$\S 2$. Es suficiente lo expuesto hasta aquí para hacer evidente la línea de argumentación que los estoicos impusieron a la ética y que, en condiciones profundamente transformadas por el nacimiento de la ciencia natural moderna, retomó Kant hace poco más de dos siglos al publicar la Fundamentación. Es esta misma distinción la que ha tendido a desaparecer por causa de la ilimitada expansión del significado del término «valor» o, en plural, «valores», que ha tenido lugar desde finales del siglo XIX hasta el presente. Curiosamente la corriente que contribuyó en mayor medida a esta inflación de su significado, fue posiblemente el Neo-kantismo del sur, especialmente H. Rickert, quien intentó fundamentar una teoría del conocimiento, en especial de la historia y de las ciencias sociales, recurriendo a la existencia de «valores objetivos» anclados en la razón práctica. Max Weber, quien siguió a Rickert en su epistemología de las ciencias sociales, dio el paso definitivo al declarar - probablemente bajo la influencia de Nietzsche- la relatividad de todos los valores, incluso los epistemológicos. Fue la sociología funcionalista de Talcott Parsons la que, recibiendo a su manera la perspectiva weberiana, terminó vaciando al término de todo significado referencial para transformarlo en un término operacional: «[u]n valor es una concepción, explícita o implícita, propia de un individuo o característica de un grupo, de la desirabilidad que influencia la selección de las formas, de los medios y de los fines de la acción» ${ }^{2}$. El relativismo actual del sentido, que puede albergar cualquier orden de preferencias en la selección de los fines de la acción, tanto individual como colectiva, es una consecuencia de este paulatino vaciamiento normativo.

Al retomar una clasificación como la propuesta por los estoicos, mi primer interés se orienta hacia una recuperación de un sentido consistente del término. La restricción del uso de preferidos y, en ese sentido, condicionalmente «valiosos», exclusivamente para aquellas acciones o estados de cosas que, siendo moralmente indiferentes, responden a una necesidad según la naturaleza, mientras que las acciones morales en sentido estricto quedan fuera y más allá tanto de las preferencias como de las inclinaciones contrarias, establece una separación radical entre lo que se debe hacer en cumplimiento de actos morales, que son fines en sí mismos y, como tales, 
incondicionados, y lo que se tiene que hacer de acuerdo con un juicio estimativo o de preferencia. En efecto, los estados de cosas valiosos son siempre condicionados y relativos, de modo que dependerán siempre de un juicio que proveerá «una justificación razonable». L. Becker, en su reciente defensa del estoicismo, define esta relación de la siguiente manera: «El entrenamiento estoico tiende a inculcarnos una fuerza motivadora categórica para los juicios normativos que se basan en una cláusula del tipo «consideradas toda las cosas», de modo que la fuerza motivadora de los juicios evaluativos de otra especie cede en situación de conflicto ante los juicios normativos [del primer tipo]» ${ }^{3}$.

A su vez, como lo muestra el texto de Estobeo, las cosas valiosas, en la medida en que dependen de juicios evaluativos, necesariamente tendrán un valor en relación con el fin o plan último que cada uno establezca para su vida. Este fin incondicionado era para el estoicismo, como para toda la ética antigua, la felicidad, aunque en este caso lo que ellos entendía bajo este término está muy lejos de lo que nosotros podemos imaginar. Quizás el mejor correlato actual para este concepto sea el de la «concepción estoica de la buena vida», ya que esta denominación más neutra se corresponde con los dos criterios necesarios y suficientes que ellos daban para esta situación: 1) actuar según la virtud, y 2) obtener la tranquilidad del alma que esto nos proporciona.

§3. Como recordé antes, algunos de los aspectos más destacables de la ética estoica fueron retomados por Kant en la elaboración de la filosofía moral que desarrolló a lo largo de su vida. A mi juicio, encontramos un claro paralelo de la distinción estoica en la división de los deberes perfectos e imperfectos que Kant establece en la Metafísica de las costumbres, sustituyendo y, de ese modo, implícitamente enmendando la que él mismo había propuesto en la Fundamentación una década atrás ${ }^{4}$. Especialmente en la «Introducción a la teoría de la virtud» (secciones VI y VII) se explaya Kant en detalle sobre el principio de esta distinción: el imperativo categórico formulado como principio del derecho exige que la acción sea de tal forma que la máxima de ella pueda valer al mismo tiempo como ley universal, de modo que el deber jurídico que de allí surge impone restricciones clara y precisamente determinadas a la acción, pero deja los fines de la máxima y, por tanto, de la acción particular librados al arbitrio del agente. Tales son los deberes perfectos ${ }^{5}$. El principio supremo de la teoría de la virtud es, en cambio, el siguiente: «actúa de acuerdo con una máxima de los fines tales que proponérselos pueda ser para cada uno una ley universal» $(M S$, p. 526). Dado que no se trata aquí de la determinación de la acción misma, sino de los fines que deben ser deberes a priori y que, por ello, no son los que naturalmente tenemos, sino los que deberíamos tener, tanto el asumirlos como tales cuanto el llevarlos efectivamente a cabo corresponde al dominio de lo subjetivo y contingente, ya que no se puede determinar por anticipado las oportunidades ni los medios para realizarlos. Éstos son, pues, los deberes puramente éticos, y, dada la indeterminación material que los afecta, deberes imperfectos o meritorios (MS, pp. 520 y ss.).

Sobre la base de esta distinción, Kant introduce las siguientes valoraciones: el cumplimiento de una acción de acuerdo con el deber jurídico es $=0$, esto es, carece de mérito o valor; la omisión de un deber de virtud, es decir, la no realización de un deber imperfecto o meritorio es también $=0$, ya que no es imputable al sujeto el no llevar a cabo actos meritorios; la realización, por último, de un deber imperfecto o de virtud conlleva un valor positivo, dado que $\alpha+0=\alpha$, con la condición, por cierto, de que la acción meritoria no sea realizada con vistas a la obtención de ese mérito, sino por la simple voluntad de 
llevar a cabo los fines a priori ${ }^{6}$. De este modo se evidencian los puntos de contacto entre la doctrina estoica y la teoría kantiana: valor moral solamente poseen los actos rectos (katórthoma) según los estoicos y las acciones realizadas en cumplimiento de deberes imperfectos de acuerdo con Kant. Los actos debidos (kathêkon) de acuerdo con los estoicos y los deberes perfectos o jurídicos (officia juris) según Kant no tienen valor moral ni positivo ni negativo, y en ese sentido constituyen el ámbito de las acciones indiferentes, en el que se abre la posibilidad de establecer órdenes de preferencia de acuerdo a los fines individuales que cada agente se proponga. Por último, los actos contrarios a los deberes perfectos tienen un disvalor moral absoluto que sólo puede ser compensado por la pena que equilibre ese disvalor, de modo que la ecuación completa dé como resultado nuevamente $0(-\alpha+\alpha=0)$.

Hace aproximadamente unos quince años Ch. Korsgaard, basándose fundamentalmente en el modo de concebir los fines últimos por parte de Aristóteles y de Kant, propuso denominar «racionalista» a una cierta concepción de los valores. La visión racionalista propuesta por ella intenta mediar entre dos posiciones tradicionales, la subjetivista, que hace depender los valores exclusivamente de los deseos, y la objetivista, que los atribuye a propiedades intrínsecas de los objetos. La teoría racionalista sostiene, en cambio, que «un objeto o estado de cosas es bueno si existe, prima facie, una razón práctica suficiente para realizarlo o producirlo» ${ }^{7}$. Tengo amplias divergencias con la reconstrucción de la filosofía práctica aristotélica que Korsgaard propone en este ensayo, y también en algunos puntos con su interpretación de la ética kantiana, la que en este trabajo se limita a los dos libros metodológicos, la Fundamentación y la Crítica de la razón práctica, dejando de lado precisamente la obra en la que Kant expone su doctrina de los fines, esto es, la Metafísica de las costumbres. Sin embargo, coincido en que la concepción racionalista de los valores, tal como ésta se presenta en la ética aristotélica de la virtud, en la teoría ética de los estoicos y en la filosofía kantiana del derecho y de la ética, todas las cuales establecen la supremacía de ciertos fines incondicionados sobre todos los demás, contingentes y sujetos al arbitrio, es la única concepción que podemos razonablemente sostener en la filosofía moral.

$\S 4$. Antes de concluir mi ponencia, me gustaría adelantarme a una posible objeción escéptica, propia de los tiempos que corren, que sería más o menos así: «Pues bien, aceptemos que la propuesta de los estoicos o de Kant, en sus propios términos, hayan sido razonables y consistentes; sin embargo, ¿de qué nos sirven a nosotros estas distinciones, nosotros que ya no podemos creer, como los estoicos, en una ley natural que gobierne al universo y la conducta de los hombres, ni, como Kant, en un derecho natural fundado en una metafísica racional a priori; nosotros, por último, para quienes la felicidad consiste, a lo sumo, en el goce efímero que nos proporciona un deseo satisfecho y que dará lugar inevitablemente en breve tiempo al dolor de un nuevo deseo insatisfecho?»

Mi respuesta, necesariamente concisa, es la siguiente: sin duda, carecemos hoy de un derecho natural, pero hemos ido recreando desde hace medio siglo un conjunto de principios morales y jurídicos considerados institucionalmente universales, que en la actualidad nadie se atreve abiertamente a rechazar ni siquiera aquellos que los violan solapadamente, los derechos humanos. Éstos se han constituido en nuestro nuevo derecho natural, que ha ido invadiendo las morales particularistas de las diversas culturas y los ordenamientos institucionales nacionales, otrora considerados soberanos, homogeneizándolos en la selección y extensión de ciertos derechos fundamentales que todos los estados se 
comprometen a garantizar. Admitiendo que los juicios basados en principios universales tienen un carácter categórico, los juicios valorativos deberían restringirse a establecer preferencias fundadas, con referencia y en relación a fines incondicionados. Por cierto, no es admisible imponer modos de buena vida a los individuos en su búsqueda de planes de vida propios, pero sí es no sólo posible, sino necesario establecer algunas de las condiciones necesarias para que cada uno esté capacitado para proponerse, proyectar y realizar su propio plan de vida autónomamente. He defendido en otra parte la tesis de que este fin constituye una concepción formal del bien, que puede ser universalmente propuesta ${ }^{8}$. Se podría objetar que una tesis como ésta conduce a alguna forma de perfeccionismo. A mi juicio, esa objeción es infundada, porque proponer condiciones generales que deben ser satisfechas para que alguien actúe autónomamente no es equivalente a dictarle a nadie cómo debe actuar, una vez alcanzada la necesaria autonomía. Sin embargo, si se me reprochara que sostener que es necesaria una educación orientada a desarrollar en cada sujeto humano sus capacidades para deliberar y actuar por sí mismos, es una forma de perfeccionismo, estoy dispuesto a admitir que se trata de un moderado ideal perfeccionista basado en las capacidades humanas, comenzando con la razón, sin el cual no existe posibilidad alguna de buena vida, al menos en los términos en que debemos entenderla dentro de una tradición como la kantiana.

\section{REFERENCIAS}

Arnim, H. V., Stoicorum Veterum Fragmenta, Stuttgart, B. G. Teubner, 1903, 4 vols.

Becker, L., A New Stoicism, Princeton Princeton U.P., 1998.

Bertomeu, M. J.; Gaeta, R., y Vidiella, G. (compiladores), Universalismo y mul- ticulturalismo, Buenos Aires, Eudeba, 2000.

Guariglia, O., Moralidad: Ética universalista y sujeto moral, Buenos Aires, Fondo de Cultura Económica, 1996.

Juliá, V.; Boeri, M., y Corso, L., Las exposiciones antiguas de la ética estoica, Buenos Aires, Eudeba, 1998.

KANT, I., «Die Metaphysik der Sitten», en, Werke in sechs Bänden, editadas por W. Weischedel, Darmstadt, Wiss. Buchgesellschaft, 1963, t. IV, pp. 303-634.

Kant's handschriflticher Nachlass, Akademie Ausgabe, t. XIX, Berlin-Leipzig, W. De Gruyter, 1934.

Kersting, W., Wohlgeordnete Freiheit, Frankfurt, Suhrkamp (2. ${ }^{\mathrm{a}}$ ed.), 1993.

- «Der kategorische Imperativ, die vollkommenen und die unvollkommenen Pflichten», Zeitschrift f. Philos. Forschung, 37 (1983), pp. 404-421.

KIDD, I. G., «Stoic Intermediates and the End for Man», en, LonG, A. A. (comp.), Problems in Stoicism, Londres, Athlone Press, 1971, pp. 150-172.

KorsgaArd, Ch., "Aristotle and Kant on the Source of Value», Ethics, 96 (1986), pp. 486-505.

Long, A. A., y Sedley, D. N., The Hellenistic Philosophers, Cambridge, CUP, 1987, 2 vols.

Parsons, T., y Shils, E. A. (comp.), Toward a General Theory of Action, New York, Harper \& Row, 1951.

Plutarco, Moralia, vol. XIII, Part II, edited with and english Translation by Harold Cherniss, Cambridge, Mass. Harvard UP, 1976.

SÉnECA, L. A., Ad Lucilium epistulae morales $-E p$ - texto editado por F. Préchac $(\mathrm{Pa}-$ ris, Les Belles Lettres, 1963-1964), traducido al alemán con notas por $\mathrm{M}$. Rosenbach, 2 t., Darmstadt, Wissenschaftliche Buchgesellschaft, 4. ${ }^{\mathrm{a}}$ ed., 1995.

Sextus Empiricus, Against the Ethicists, ed. and translated by R. G. Bury, Londres-Cambridge, Harvard UP, 1960 , vol. III.

White, N., «Stoic Values», The Monist, 73 (1990), pp. 42-58. 


\section{NOTAS}

${ }_{1} \mathrm{El}$ tema de los indiferentes o intermediarios es especialmente controvertido. La interpretación que he ofrecido sigue de cerca la propuesta por Kidd, 1971, pp. 155 ss. Sobre toda la relación entre la estimabilidad de los indiferentes y la virtud, es importante la discusión de White, 1990, pp. 43 y ss.

2 Parsons y Shils, 1951, p. 395.

${ }^{3}$ Becker, 1998, p. 14.

${ }^{4}$ Cp. Kersting, 1983, pp. 404 y ss., y 1993, pp. 187 ss.
${ }^{5} M S$, pp. 519-520 (Weischedel).

${ }^{6}$ Cp. Kant, XIX, p. 96, Ref. 6585; p. 261, Ref. 7165; MS, p. 520 (Weischedel); Kersting, 1993, p. 186.

${ }^{7}$ Korsgaard, 1986, p. 487.

${ }^{8}$ Cp. Guariglia, 1996, pp. 187 y ss. Al respecto véanse ahora las discusiones de Ferraro, pp. 255 y ss. y de Bertomeu y Vidiella, pp. 297 y ss., ambas en Bertomeu, Gaeta y Vidiella (comp.), 2000. 